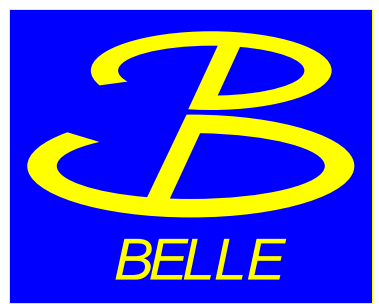

KEK Preprint 2002-6

Belle Preprint 2002-8

\title{
Study of $\boldsymbol{C P}$-Violating Asymmetries in $B^{0} \rightarrow \pi^{+} \pi^{-}$Decays
}

K. Abe,${ }^{9}$ K. Abe, ${ }^{44}$ T. Abe,${ }^{45}$ I. Adachi, ${ }^{9}$ Byoung Sup Ahn, ${ }^{17}$ H. Aihara, ${ }^{46}$ M. Akatsu, ${ }^{24}$ Y. Asano,${ }^{51}$ T. Aso,${ }^{50}$ V. Aulchenko, ${ }^{2}$ T. Aushev ${ }^{14}$ A. M. Bakich, ${ }^{41}$ Y. Ban,${ }^{35}$ E. Banas,${ }^{29}$ I. Bedny, ${ }^{2}$ S. Behari,${ }^{9}$ P. K. Behera,${ }^{52}$ A. Bondar,${ }^{2}$ A. Bozek, ${ }^{29}$ M. Bračko, ${ }^{22,15}$ J. Brodzicka, ${ }^{29}$ T. E. Browder ${ }^{8}$ B. C. K. Casey,${ }^{8}$ P. Chang, ${ }^{28}$ Y. Chao, ${ }^{28}$ B. G. Cheon,${ }^{40}$ R. Chistov ${ }^{14}$ S.-K. Choi, ${ }^{7}$ Y. Choi,${ }^{40}$ M. Danilov, ${ }^{14}$ L. Y. Dong, ${ }^{12}$ J. Dragic ${ }^{23}$ A. Drutskoy,${ }^{14}$ S. Eidelman,${ }^{2}$ V. Eiges,${ }^{14}$ Y. Enari,${ }^{24}$ C. W. Everton, ${ }^{23}$ F. Fang, ${ }^{8}$ H. Fujii,${ }^{9}$ C. Fukunaga,${ }^{48}$ M. Fukushima, ${ }^{11}$ N. Gabyshev,${ }^{9}$ A. Garmash, ${ }^{2,9}$ T. Gershon, ${ }^{9}$ B. Golob,${ }^{21,15}$ A. Gordon, ${ }^{23}$ R. Guo, ${ }^{26}$ J. Haba,${ }^{9}$ H. Hamasaki,${ }^{9}$ K. Hanagaki,${ }^{36}$ F. Handa,${ }^{45}$ K. Hara,${ }^{33}$ T. Hara,${ }^{33}$ N. C. Hastings, ${ }^{23}$ H. Hayashii, ${ }^{25}$ M. Hazumi,${ }^{9}$ E. M. Heenan,${ }^{23}$ I. Higuchi, ${ }^{45}$ T. Higuchi, ${ }^{46}$ T. Hojo, ${ }^{33}$ T. Hokuue, ${ }^{24}$ Y. Hoshi, ${ }^{44}$ K. Hoshina, ${ }^{49}$ S. R. Hou ${ }^{28}$ W.-S. Hou, ${ }^{28}$ S.-C. Hsu, ${ }^{28}$ H.-C. Huang,${ }^{28}$ T. Igaki, ${ }^{24}$ Y. Igarashi,${ }^{9}$ T. Iijima, ${ }^{9}$ H. Ikeda,${ }^{9}$ K. Inami, ${ }^{24}$ A. Ishikawa, ${ }^{24}$ H. Ishino,,${ }^{47}$ R. Itoh,${ }^{9}$ M. Iwamoto, ${ }^{3}$ H. Iwasaki,${ }^{9}$ Y. Iwasaki,${ }^{9}$ D. J. Jackson, ${ }^{33}$ P. Jalocha, ${ }^{29}$ H. K. Jang, ${ }^{39}$ J. H. Kang, ${ }^{55}$ J. S. Kang, ${ }^{17}$ P. Kapusta, ${ }^{29}$ N. Katayama, ${ }^{9}$ H. Kawai, ${ }^{3}$ H. Kawai, ${ }^{46}$ N. Kawamura, ${ }^{1}$ T. Kawasaki,${ }^{31}$ H. Kichimi, ${ }^{9}$ D. W. Kim ${ }^{40}$ Heejong Kim ${ }^{55}$ H. J. Kim ${ }^{55}$ H. O. Kim ${ }^{40}$ Hyunwoo Kim ${ }^{17}$ S. K. Kim ${ }^{39}$ T. H. Kim,${ }^{55}$ K. Kinoshita, ${ }^{5}$ S. Koishi, ${ }^{47}$ H. Konishi, ${ }^{49}$ S. Korpar, ${ }^{22,15}$ P. Križan,${ }^{21,15}$ P. Krokovny, ${ }^{2}$ R. Kulasiri, ${ }^{5}$ S. Kumar, ${ }^{34}$ A. Kuzmin, ${ }^{2}$ Y.-J. Kwon, ${ }^{55}$ J. S. Lange, ${ }^{6,37}$ G. Leder ${ }^{13}$ S. H. Lee ${ }^{39}$ A. Limosani, ${ }^{23}$ D. Liventsev, ${ }^{14}$ R.-S. Lu, ${ }^{28}$ J. MacNaughton,,${ }^{13}$ G. Majumder, ${ }^{42}$ F. Mandl,${ }^{13}$ D. Marlow ${ }^{36}$ T. Matsuishi, ${ }^{24}$ S. Matsumoto, ${ }^{4}$ T. Matsumoto, ${ }^{24}$ Y. Mikami, ${ }^{45}$ W. Mitaroff, ${ }^{13}$ K. Miyabayashi, ${ }^{25}$ Y. Miyabayashi, ${ }^{24}$ H. Miyake, ${ }^{33}$ H. Miyata ${ }^{31}$ G. R. Moloney,${ }^{23}$ S. Mori,${ }^{51}$ T. Mori,${ }^{4}$ A. Murakami,${ }^{38}$ T. Nagamine,,${ }^{45}$ Y. Nagasaka, ${ }^{10}$ T. Nakadaira, ${ }^{46}$ E. Nakano, ${ }^{32}$ M. Nakao, ${ }^{9}$ J. W. Nam, ${ }^{40}$ K. Neichi, ${ }^{44}$ S. Nishida ${ }^{18}$ O. Nitoh ${ }^{49}$ S. Noguchi,${ }^{25}$ T. Nozaki, ${ }^{9}$ S. Ogawa ${ }^{43}$ F. Ohno, ${ }^{47}$ T. Ohshima,${ }^{24}$ T. Okabe, ${ }^{24}$ S. Okuno, ${ }^{16}$ S. L. Olsen,${ }^{8}$ W. Ostrowicz,${ }^{29}$ H. Ozaki, ${ }^{9}$ P. Pakhlov, ${ }^{14}$ H. Palka, ${ }^{29}$ C. W. Park,${ }^{17}$ H. Park, ${ }^{19}$ K. S. Park, ${ }^{40}$ L. S. Peak ${ }^{41}$ J.-P. Perroud,${ }^{20}$ M. Peters ${ }^{8}$ L. E. Piilonen, ${ }^{53}$ E. Prebys, ${ }^{36}$ J. L. Rodriguez,${ }^{8}$ F. J. Ronga, ${ }^{20}$ M. Rozanska, ${ }^{29}$ K. Rybicki, ${ }^{29}$ H. Sagawa, ${ }^{9}$ S. Saitoh,${ }^{3}$ Y. Sakai, ${ }^{9}$ H. Sakamoto, ${ }^{18}$ M. Satapathy,${ }^{52}$ A. Satpathy,${ }^{9,5}$ O. Schneider,${ }^{20}$ S. Schrenk, ${ }^{5}$ C. Schwanda, ${ }^{9,13}$ S. Semenov ${ }^{14}$ K. Senyo, ${ }^{24}$ M. E. Sevior ${ }^{23}$ H. Shibuya ${ }^{43}$ B. Shwartz, ${ }^{2}$ V. Sidorov, ${ }^{2}$ J. B. Singh ${ }^{34}$ S. Stanič,${ }^{51, \text { 冈 }}$ A. Sugi, ${ }^{24}$ A. Sugiyama, ${ }^{24}$ K. Sumisawa,${ }^{9}$ T. Sumiyoshi, ${ }^{9}$ K. Suzuki,${ }^{9}$ S. Suzuki,${ }^{54}$ S. Y. Suzuki, ${ }^{9}$ S. K. Swain, ${ }^{8}$ H. Tajima ${ }^{46}$ T. Takahashi, ${ }^{32}$ F. Takasaki, ${ }^{9}$ M. Takita,${ }^{33}$ K. Tamai, ${ }^{9}$ N. Tamura, ${ }^{31}$ J. Tanaka,${ }^{46}$ M. Tanaka,${ }^{9}$ G. N. Taylor, ${ }^{23}$ Y. Teramoto,${ }^{32}$ S. Tokuda,${ }^{24}$ M. Tomoto,${ }^{9}$ T. Tomura,${ }^{46}$ S. N. Tovey ${ }^{23}$ K. Trabelsi, ${ }^{8}$ W. Trischuk ${ }^{36,}$, T. Tsuboyama, ${ }^{9}$ T. Tsukamoto, ${ }^{9}$ S. Uehara,${ }^{9}$ K. Ueno,${ }^{28}$ Y. Unno, ${ }^{3}$ S. Uno,${ }^{9}$ Y. Ushiroda,${ }^{9}$ K. E. Varvell, ${ }^{41}$ C. C. Wang, ${ }^{28}$ C. H. Wang, ${ }^{27}$ J. G. Wang, ${ }^{53}$ M.-Z. Wang ${ }^{28}{ }^{28}$ Y. Watanabe,${ }^{47}$ E. Won ${ }^{39}$ B. D. Yabsley,${ }^{9}$ Y. Yamada,${ }^{9}$ M. Yamaga,${ }^{45}$ A. Yamaguchi ${ }^{45}$ H. Yamamoto,${ }^{45}$ Y. Yamashita ${ }^{30}$ M. Yamauchi, ${ }^{9}$ J. Yashima,${ }^{9}$ P. Yeh,${ }^{28}$ M. Yokoyama,${ }^{46} \mathrm{~K}$. Yoshida,${ }^{24}$ Y. Yuan, ${ }^{12}$ Y. Yusa, ${ }^{45}$ C. C. Zhang, ${ }^{12}$ J. Zhang, ${ }^{51}$ Y. Zheng, ${ }^{8}$ V. Zhilich, ${ }^{2}$ and D. Žontar ${ }^{51}$ 
(The Belle Collaboration)

${ }^{1}$ Aomori University, Aomori

${ }^{2}$ Budker Institute of Nuclear Physics, Novosibirsk

${ }^{3}$ Chiba University, Chiba

${ }^{4}$ Chuo University, Tokyo

${ }^{5}$ University of Cincinnati, Cincinnati $\mathrm{OH}$

${ }^{6}$ University of Frankfurt, Frankfurt

${ }^{7}$ Gyeongsang National University, Chinju

${ }^{8}$ University of Hawaii, Honolulu HI

${ }^{9}$ High Energy Accelerator Research Organization (KEK), Tsukuba

${ }^{10}$ Hiroshima Institute of Technology, Hiroshima

${ }^{11}$ Institute for Cosmic Ray Research, University of Tokyo, Tokyo

${ }^{12}$ Institute of High Energy Physics,

Chinese Academy of Sciences, Beijing

${ }^{13}$ Institute of High Energy Physics, Vienna

${ }^{14}$ Institute for Theoretical and Experimental Physics, Moscow

${ }^{15} \mathrm{~J}$. Stefan Institute, Ljubljana

${ }^{16}$ Kanagawa University, Yokohama

${ }^{17}$ Korea University, Seoul

${ }^{18}$ Kyoto University, Kyoto

${ }^{19}$ Kyungpook National University, Taegu

${ }^{20}$ IPHE, University of Lausanne, Lausanne

${ }^{21}$ University of Ljubljana, Ljubljana

${ }^{22}$ University of Maribor, Maribor

${ }^{23}$ University of Melbourne, Victoria

${ }^{24}$ Nagoya University, Nagoya

${ }^{25}$ Nara Women's University, Nara

${ }^{26}$ National Kaohsiung Normal University, Kaohsiung

${ }^{27}$ National Lien-Ho Institute of Technology, Miao Li

${ }^{28}$ National Taiwan University, Taipei

${ }^{29}$ H. Niewodniczanski Institute of Nuclear Physics, Krakow

${ }^{30}$ Nihon Dental College, Niigata

${ }^{31}$ Niigata University, Niigata

${ }^{32}$ Osaka City University, Osaka

${ }^{33}$ Osaka University, Osaka

${ }^{34}$ Panjab University, Chandigarh

${ }^{35}$ Peking University, Beijing

${ }^{36}$ Princeton University, Princeton NJ

${ }^{37}$ RIKEN BNL Research Center, Brookhaven NY

${ }^{38}$ Saga University, Saga

${ }^{39}$ Seoul National University, Seoul

${ }^{40}$ Sungkyunkwan University, Suwon

${ }^{41}$ University of Sydney, Sydney NSW

${ }^{42}$ Tata Institute of Fundamental Research, Bombay

${ }^{43}$ Toho University, Funabashi

44 Tohoku Gakuin University, Tagajo

${ }^{45}$ Tohoku University, Sendai 


$$
\begin{gathered}
{ }^{46} \text { University of Tokyo, Tokyo } \\
{ }^{47} \text { Tokyo Institute of Technology, Tokyo } \\
{ }^{48} \text { Tokyo Metropolitan University, Tokyo } \\
{ }^{49} \text { Tokyo University of Agriculture and Technology, Tokyo } \\
{ }^{50} \text { Toyama National College of Maritime Technology, Toyama } \\
{ }^{51} \text { University of Tsukuba, Tsukuba } \\
{ }^{52} \text { Utkal University, Bhubaneswer } \\
{ }^{53} \text { Virginia Polytechnic Institute and State University, Blacksburg VA } \\
{ }^{54} \text { Yokkaichi University, Yokkaichi } \\
\text { 55 Yonsei University, Seoul }
\end{gathered}
$$

(Dated: October 24, 2018)

\begin{abstract}
We present a measurement of $C P$-violating asymmetries in $B^{0} \rightarrow \pi^{+} \pi^{-}$decays based on a $41.8 \mathrm{fb}^{-1}$ data sample collected at the $\Upsilon(4 S)$ resonance with the Belle detector at the KEKB asymmetric-energy $e^{+} e^{-}$collider. We fully reconstruct one neutral $B$ meson as a $B^{0} \rightarrow \pi^{+} \pi^{-}$ $C P$ eigenstate and identify the flavor of the accompanying $B$ meson from its decay products. From the asymmetry in the distribution of the time intervals between the two $B$ meson decay points, we obtain the $C P$-violating asymmetry parameters $\mathcal{S}_{\pi \pi}=-1.21_{-0.27}^{+0.38}$ (stat) ${ }_{-0.13}^{+0.16}$ (syst) and $\mathcal{A}_{\pi \pi}=+0.94_{-0.31}^{+0.25}$ (stat) \pm 0.09 (syst).
\end{abstract}

PACS numbers: PACS numbers: 11.30.Er, 12.15.Hh, 13.25.Hw 
Kobayashi and Maskawa (KM) proposed, in 1973, a model where $C P$ violation is incorporated as an irreducible complex phase in the weak-interaction quark mixing matrix [1]. Recent measurements of the $C P$-violating parameter $\sin 2 \phi_{1}$ by the Belle [2] and BaBar [3] collaborations established $C P$ violation in the neutral $B$ meson system that is consistent with KM expectations. Measurements of other $C P$-violating parameters provide important tests of the KM model. In this Letter we describe a measurement of $C P$-violating asymmetries in the mode $B^{0} \rightarrow \pi^{+} \pi^{-}$[4]; these are sensitive to the parameter sin $2 \phi_{2}$ [5].

The KM model predicts $C P$-violating asymmetries in the time-dependent rates for $B^{0}$ and $\bar{B}^{0}$ decays to a common $C P$ eigenstate, $f_{C P}$ [6]. In the decay chain $\Upsilon(4 S) \rightarrow B^{0} \bar{B}^{0} \rightarrow$ $f_{C P} f_{\mathrm{tag}}$, where one of $B$ mesons decays at time $t_{C P}$ to $f_{C P}$ and the other decays at time $t_{\text {tag }}$ to a final state $f_{\text {tag }}$ that distinguishes between $B^{0}$ and $\bar{B}^{0}$, the decay rate has a time dependence given by [7]

$$
\begin{aligned}
\mathcal{P}_{\pi \pi}^{q}(\Delta t)=\frac{e^{-|\Delta t| / \tau_{B^{0}}}}{4 \tau_{B^{0}}}[1+ & q \cdot\left\{\mathcal{S}_{\pi \pi} \sin \left(\Delta m_{d} \Delta t\right)\right. \\
& \left.\left.+\mathcal{A}_{\pi \pi} \cos \left(\Delta m_{d} \Delta t\right)\right\}\right],
\end{aligned}
$$

where $\tau_{B^{0}}$ is the $B^{0}$ lifetime, $\Delta m_{d}$ is the mass difference between the two $B^{0}$ mass eigenstates, $\Delta t=t_{C P}-t_{\mathrm{tag}}$, and the $b$-flavor charge $q=+1(-1)$ when the tagging $B$ meson is a $B^{0}\left(\bar{B}^{0}\right)$. The $C P$-violating parameters $\mathcal{S}_{\pi \pi}$ and $\mathcal{A}_{\pi \pi}$ defined in Eq. (1) are expressed by $\mathcal{S}_{\pi \pi}=2 \operatorname{Im} \lambda /\left(|\lambda|^{2}+1\right)$ and $\mathcal{A}_{\pi \pi}=\left(|\lambda|^{2}-1\right) /\left(|\lambda|^{2}+1\right)$, where $\lambda$ is a complex parameter that depends on both $B^{0} \bar{B}^{0}$ mixing and on the amplitudes for $B^{0}$ and $\bar{B}^{0}$ decay to $\pi^{+} \pi^{-}$. In the $\mathrm{SM}$, to a good approximation, $|\lambda|$ is equal to the absolute value of the ratio of the $\bar{B}^{0}$ to $B^{0}$ decay amplitudes. We would have $\mathcal{S}_{\pi \pi}=\sin 2 \phi_{2}$ and $\mathcal{A}_{\pi \pi}=0$, or equivalently $|\lambda|=1$, if the $b \rightarrow u$ tree amplitude were dominant. The situation is complicated by the possibility of significant contributions from gluonic $b \rightarrow d$ penguin amplitudes that have a different weak phase and additional strong phases [8]. As a result, $\mathcal{S}_{\pi \pi}$ may not be equal to $\sin 2 \phi_{2}$ and direct $C P$ violation, $\mathcal{A}_{\pi \pi} \neq 0$, may occur.

This measurement is based on a $41.8 \mathrm{fb}^{-1}$ data sample, which contains 44.8 million $B \bar{B}$ pairs, collected with the Belle detector at the KEKB asymmetric-energy $e^{+} e^{-}(3.5$ on $8 \mathrm{GeV}$ ) collider [9] operating at the $\Upsilon(4 S)$ resonance. At KEKB, the $\Upsilon(4 S)$ is produced with a Lorentz boost of $\beta \gamma=0.425$ nearly along the electron beamline $(z)$. Since the $B^{0}$ and $\bar{B}^{0}$ mesons are approximately at rest in the $\Upsilon(4 S)$ center-of-mass system (cms), $\Delta t$ can be determined from the displacement in $z$ between the $f_{C P}$ and $f_{\text {tag }}$ decay vertices: $\Delta t \simeq\left(z_{C P}-z_{\mathrm{tag}}\right) / \beta \gamma c \equiv \Delta z / \beta \gamma c$.

The Belle detector [10 is a large-solid-angle spectrometer that consists of a silicon vertex detector (SVD), a central drift chamber (CDC), an array of aerogel threshold Čerenkov counters (ACC), time-of-flight scintillation counters (TOF), and an electromagnetic calorimeter comprised of $\mathrm{CsI}(\mathrm{Tl})$ crystals (ECL) located inside a super-conducting solenoid coil that provides a $1.5 \mathrm{~T}$ magnetic field. An iron flux-return located outside of the coil is instrumented to detect $K_{L}^{0}$ mesons and to identify muons (KLM).

The $B^{0} \rightarrow \pi^{+} \pi^{-}$event selection is described in detail elsewhere [11]. We use oppositely charged track pairs that are positively identified as pions according to the combined information from the ACC and the CDC $d E / d x$ measurement. Candidate $B$ mesons are reconstructed using the energy difference $\Delta E \equiv E_{B}^{\mathrm{cms}}-E_{\text {beam }}^{\mathrm{cms}}$ and the beam-energy constrained mass $M_{\mathrm{bc}} \equiv \sqrt{\left(E_{\mathrm{beam}}^{\mathrm{cms}}\right)^{2}-\left(p_{B}^{\mathrm{cms}}\right)^{2}}$, where $E_{\mathrm{beam}}^{\mathrm{cms}}$ is the cms beam energy, and $E_{B}^{\mathrm{cms}}$ and $p_{B}^{\mathrm{cms}}$ are the cms energy and momentum of the $B$ candidate. The signal region is defined as 5.271 $<M_{\mathrm{bc}}<5.287 \mathrm{GeV} / c^{2}$ and $|\Delta E|<0.067 \mathrm{GeV}$, corresponding to $\pm 3 \sigma$ from the central 
values. In order to suppress background from the $e^{+} e^{-} \rightarrow q \bar{q}$ continuum $(q=u, d, s, c)$, we form signal and background likelihood functions, $\mathcal{L}_{S}$ and $\mathcal{L}_{B G}$, from two variables. One is a Fisher discriminant determined from six modified Fox-Wolfram moments |12]; the other is the $B$ flight direction in the cms, with respect to the $z$ axis $\left(\cos \theta_{B}\right)$. We determine $\mathcal{L}_{S}$ from Monte Carlo $(\mathrm{MC})$ and $\mathcal{L}_{B G}$ from data, and require $\mathcal{L}_{S} /\left(\mathcal{L}_{S}+\mathcal{L}_{B G}\right)>0.825$ for candidate events. Figure 1 shows the $\Delta E$ distribution for $\pi^{+} \pi^{-}$candidates. The signal

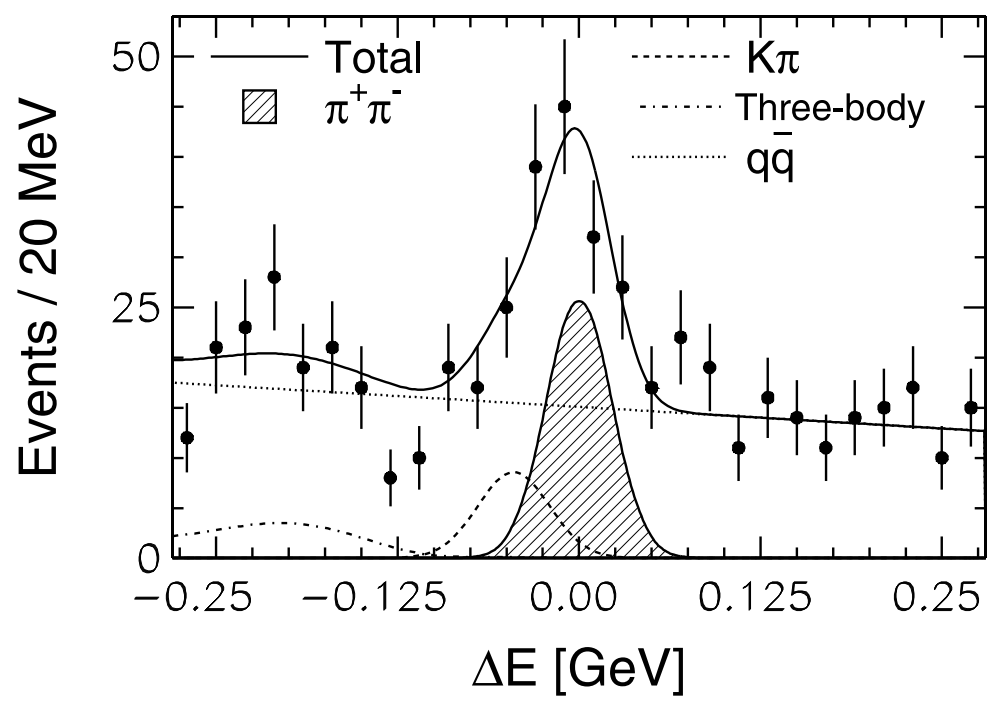

FIG. 1: $\Delta E$ distribution for $\pi^{+} \pi^{-}$event candidates that are in the $M_{\mathrm{bc}}$ signal region.

yield is extracted by fitting the $\Delta E$ distribution with a Gaussian $\pi^{+} \pi^{-}$signal function, plus contributions from misidentified $B^{0} \rightarrow K^{+} \pi^{-}$events, three-body $B$-decays, and continuum background. From the fit, we obtain $73.5 \pm 13.8$ (stat) $\pi^{+} \pi^{-}$events, $28.4 \pm 12.5$ (stat) $K^{+} \pi^{-}$ events, and $98.7 \pm 7.0$ (stat) continuum events in the signal region. The $K^{+} \pi^{-}$contamination is consistent with the $K \rightarrow \pi$ misidentification probability measured independently. The contribution from three-body $B$-decays is negligibly small in the signal region.

Leptons, charged pions, and kaons that are not associated with the reconstructed $B^{0} \rightarrow$ $\pi^{+} \pi^{-}$decay are used to identify the flavor of the accompanying $B$ meson. We use two parameters, $q$ and $r$, to represent the tagging information. The first, $q$, is already defined in Eq. (1). The parameter $r$ is an event-by-event, MC-determined flavor-tagging dilution factor that ranges from $r=0$ for no flavor discrimination to $r=1$ for unambiguous flavor assignment. It is used only to sort data into six $r$ intervals (boundaries at $0.25,0.5,0.625$, 0.75 and 0.875$)$. The wrong tag fractions for the six $r$ intervals, $w_{l}(l=1,6)$, are determined from the data; we use the same $w_{l}$ values that were used for the $\sin 2 \phi_{1}$ measurement [2].

The vertex positions for the $\pi^{+} \pi^{-}$and $f_{\text {tag }}$ decays are reconstructed using tracks with associated hits in the SVD. Each vertex position is also constrained by the interaction point profile smeared in the $r$ - $\phi$ plane by the average transverse $B$ meson decay length. The $f_{\text {tag }}$ vertex is determined from all well reconstructed tracks, excluding the $B^{0} \rightarrow \pi^{+} \pi^{-}$candidate and tracks that form a $K_{S}^{0}$ candidate.

The proper-time interval resolution for the signal, $R_{\text {sig }}(\Delta t)$, is obtained by convolving a sum of two Gaussians (a main component, plus a tail component caused by poorly reconstructed tracks) with a function that takes into account the cms motion of the $B$ mesons. We use the same parameters as those used for the $\sin 2 \phi_{1}$ measurement; the fraction and 
the typical width of the main Gaussian are $0.97 \pm 0.02$ and 1.49 ps, respectively [2]. The background resolution function $R_{q \bar{q}}(\Delta t)$, which is dominated by continuum background, has the same functional form but the parameters are obtained from a sideband region in $M_{b c}$ and $\Delta E$. Using these resolution functions, we perform a $B^{0}$ lifetime measurement that yields $\tau_{B^{0}}=1.49 \pm 0.21$ (stat) ps for $B^{0} \rightarrow \pi^{+} \pi^{-}$candidates, which is consistent with the world average value 13 .

We determine $C P$ violation parameters by performing an unbinned maximum-likelihood fit of a $C P$-violating probability density function (pdf) to the $\Delta t$ distributions. We define the likelihood value for each event as a function of $\mathcal{S}_{\pi \pi}$ and $\mathcal{A}_{\pi \pi}$ :

$$
\begin{aligned}
P_{i}=\int\left[\left\{f_{\pi \pi}^{l} \mathcal{P}_{\pi \pi}^{q}\left(\Delta t^{\prime}, w_{l} ; \mathcal{S}_{\pi \pi}, \mathcal{A}_{\pi \pi}\right)+\right.\right. & \left.f_{K \pi}^{l} \mathcal{P}_{K \pi}^{q}\left(\Delta t^{\prime}, w_{l}\right)\right\} \cdot R_{\mathrm{sig}}\left(\Delta t_{i}-\Delta t^{\prime}\right) \\
& \left.+f_{q \bar{q}}^{l} \mathcal{P}_{q \bar{q}}\left(\Delta t^{\prime}\right) \cdot R_{q \bar{q}}\left(\Delta t_{i}-\Delta t^{\prime}\right)\right] d \Delta t^{\prime}
\end{aligned}
$$

Here $f_{\pi \pi}^{l}, f_{K \pi}^{l}$, and $f_{q \bar{q}}^{l}\left(=1-f_{\pi \pi}^{l}-f_{K \pi}^{l}\right)$ are the fractions of $\pi^{+} \pi^{-}$signal, $K^{+} \pi^{-}$background, and continuum background in flavor-tagging interval $l$, respectively. These fractions are determined on an event-by-event basis as a function of $\Delta E$ and $M_{\mathrm{bc}}$, properly normalized by the average signal and background fractions in the signal region. The average fractions of $q \bar{q}$ background for six $r$ bins $(l=1,6)$ are $0.632,0.505,0.462,0.440,0.322$ and 0.117. For higher $r$ values where we are more sensitive to the asymmetry, the fraction of continuum background decreases; the ratio of $\pi^{+} \pi^{-}$signal events to background $K^{+} \pi^{-}$events is the same for all $r$ bins. The pdfs for $\pi^{+} \pi^{-}\left(\mathcal{P}_{\pi \pi}^{q}\right), K^{+} \pi^{-}\left(\mathcal{P}_{K \pi}^{q}\right)$, and continuum background $\left(\mathcal{P}_{q \bar{q}}\right)$, are convolved with their respective resolution functions. We use the same vertex resolution function for $\pi^{+} \pi^{-}$and $K^{+} \pi^{-}$candidates. For the $\pi^{+} \pi^{-}$signal, the pdf is given by Eq. (1) with $q$ replaced by $q\left(1-2 w_{l}\right)$, to account for the dilution due to wrong flavor tagging. The pdf for the $K^{+} \pi^{-}$background is $\mathcal{P}_{K \pi}^{q}\left(\Delta t, w_{l}\right)=e^{-|\Delta t| / \tau_{B^{0}}} / 4 \tau_{B^{0}}\left\{1+q \cdot\left(1-2 w_{l}\right) \mathcal{A}_{K \pi} \cos \left(\Delta m_{d} \Delta t\right)\right\}$, where $\mathcal{A}_{K \pi}$ is the $\bar{B}^{0} \rightarrow K^{-} \pi^{+}$and $B^{0} \rightarrow K^{+} \pi^{-}$decay rate asymmetry. We fix $\mathcal{A}_{K \pi}=0$ [11], and $\tau_{B^{0}}$ and $\Delta m_{d}$ to their world average values [13]. The pdf used for the $q \bar{q}$ background is $\mathcal{P}_{q \bar{q}}(\Delta t)=\left\{f_{\tau} e^{-|\Delta t| / \tau_{\mathrm{bkg}}} / 2 \tau_{\mathrm{bkg}}+\left(1-f_{\tau}\right) \delta(\Delta t)\right\} / 2$, where $f_{\tau}$ is the background fraction with an effective lifetime $\tau_{\mathrm{bkg}}$ and $\delta$ is the Dirac delta function. We determine $f_{\tau}=0.011 \pm 0.004$ and $\tau_{\mathrm{bkg}}=2.7_{-0.7}^{+1.0} \mathrm{ps}$ from the sideband data. In the fit, $\mathcal{S}_{\pi \pi}$ and $\mathcal{A}_{\pi \pi}$ are free parameters determined by maximizing the likelihood function $\mathcal{L}=\prod_{i} P_{i}$, where the product is over all $B^{0} \rightarrow \pi^{+} \pi^{-}$candidates.

The result of the fit to the 162 candidates $\left(92 B^{0}\right.$ - and $70 \bar{B}^{0}$-tags $)$ that remain after flavor tagging and vertex reconstruction is:

$$
\begin{aligned}
& \mathcal{S}_{\pi \pi}=-1.21_{-0.27}^{+0.38}(\text { stat }){ }_{-0.13}^{+0.16}(\text { syst }) \\
& \mathcal{A}_{\pi \pi}=+0.94_{-0.31}^{+0.25}(\text { stat }) \pm 0.09 \text { (syst) } .
\end{aligned}
$$

The result is $1.3 \sigma$ away from the physical boundary $\mathcal{S}_{\pi \pi}^{2}+\mathcal{A}_{\pi \pi}^{2}=1$, which is consistent with a statistical fluctuation. The correlation between $\mathcal{S}_{\pi \pi}$ and $\mathcal{A}_{\pi \pi}$ is found to be 0.28 . In Figs. 2(a) and (b), we show the $\Delta t$ distributions for $B^{0}$ - and $\bar{B}^{0}$-tagged events together with the fit curves; the background-subtracted $\Delta t$ distributions are shown in Fig. 2(c). Figure $2(d)$ shows the background-subtracted $C P$ asymmetry between the $B^{0}$ - and $\bar{B}^{0}$-tagged events as a function of $\Delta t$. The result of the fit is superimposed and shown by the solid curve.

The systematic error on $\mathcal{S}_{\pi \pi}$ is primarily due to uncertainties in the background fractions $( \pm 0.09)$ and a possible fit bias near the physical boundary $\left({ }_{-0.02}^{+0.11}\right)$. For $\mathcal{A}_{\pi \pi}$, the background fractions $( \pm 0.06)$ and the wrong-tag fractions $( \pm 0.06)$ are the two leading components. We 


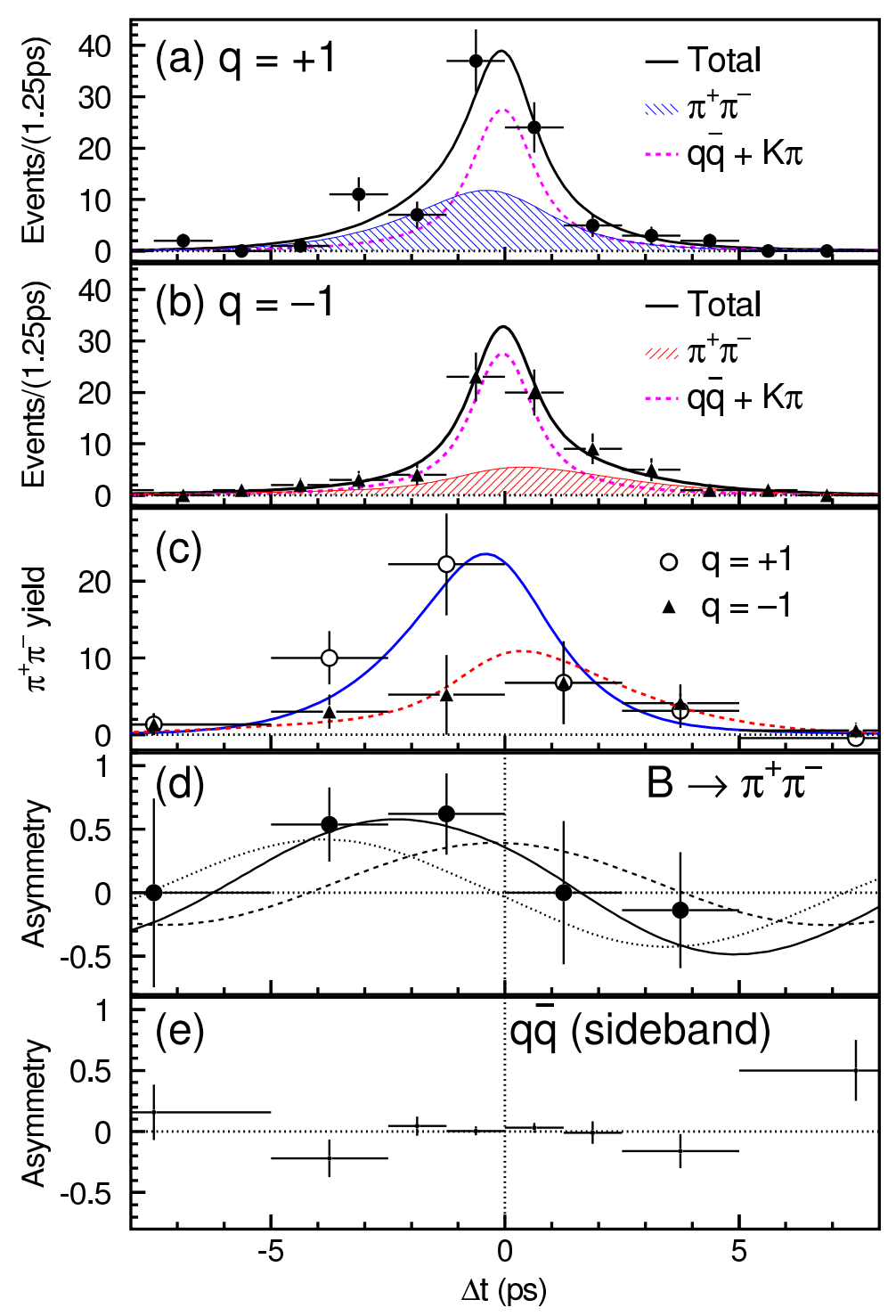

FIG. 2: The $\Delta t$ distributions for the $B^{0} \rightarrow \pi^{+} \pi^{-}$candidates in the signal region: (a) candidates with $q=+1$, i.e. the tag side is identified as $B^{0}$; (b) candidates with $q=-1$; (c) $\pi^{+} \pi^{-}$yields after background subtraction. The errors are statistical only and do not include the error of the subtracted background obtained by a fit. The rightmost (leftmost) bin ranges from 5 to 10 ps $(-5$ to $-10 \mathrm{ps}$ ); (d) the $C P$ asymmetry for $B^{0} \rightarrow \pi^{+} \pi^{-}$after background subtraction. The point in the rightmost bin has a large negative value that is outside of the range of the histogram; (e) the raw asymmetry for $B^{0} \rightarrow \pi^{+} \pi^{-}$sideband events. In Figs. (a) through (c), the curves show the results of the unbinned maximum likelihood fit. In Fig. (d), the solid curve shows the resultant $C P$ asymmetry, while the dashed (dotted) curve is the contribution from the cosine (sine) term.

find that uncertainties in $f_{\pi \pi}^{l}, f_{K \pi}^{l}$ and $f_{q \bar{q}}^{l}$ in Eq. (2) account for the largest systematic errors. We add their contributions in quadrature to obtain the above values, where each contribution is obtained by varying a parameter by its error and repeating the fit. Other sources of systematic error are uncertainties in the resolution function, physics parameters $\left(\Delta m_{d}, \tau_{B^{0}}\right.$, and $\left.\mathcal{A}_{K \pi}\right)$ and the background modeling. A value of $\mathcal{A}_{K \pi}=-0.06 \pm 0.08$ is 
obtained from the self-tagged $B^{0} \rightarrow K^{+} \pi^{-}$sample [11; this introduces a systematic error of $<0.01$ for $\mathcal{S}_{\pi \pi}$ and ${ }_{-0.01}^{+0.02}$ for $\mathcal{A}_{\pi \pi}$.

We validate our fitting procedure using a large ensemble of MC pseudo-experiments wherein events are generated with nominal pdfs and the observed number of events. For various input values of $\mathcal{S}_{\pi \pi}$ and $\mathcal{A}_{\pi \pi}$, we confirm that there is no bias in the fit results. We find that the average expected errors, 0.52 for $\mathcal{S}_{\pi \pi}$ and 0.35 for $\mathcal{A}_{\pi \pi}$, are larger than our measurements. However, the probability of obtaining errors that are smaller than our measurement is $5.4 \%$ for $\mathcal{S}_{\pi \pi}$ and $23.6 \%$ for $\mathcal{A}_{\pi \pi}$; the results are within the expected range of statistical fluctuations. The measured likelihood is in good agreement with the average likelihood value obtained in the pseudo-experiments.

We perform a number of cross checks. We examine the event yields and $\Delta t$ distributions for $B^{0}$ - and $\bar{B}^{0}$-tagged events in the sideband region and find no significant asymmetry as shown in Fig. 2(e). We select $B^{0} \rightarrow K^{+} \pi^{-}$candidates, which have the same track topology as $B^{0} \rightarrow \pi^{+} \pi^{-}$, by positively identifying charged kaons. A fit to 309 candidates $(209 \pm 16$ signal events) yields $\tau_{B^{0}}=1.73 \pm 0.15$ (stat) ps and $\Delta m_{d}=0.57 \pm 0.08$ (stat) $\mathrm{ps}^{-1}$; these are consistent with the world average values [13]. $\mathcal{A}_{K \pi}$ is $0.07 \pm 0.17$, in agreement with the counting analysis mentioned above and $\mathcal{S}_{K \pi}=0.15 \pm 0.24$, which is consistent with zero. We also select $B^{0} \rightarrow D^{-} \pi^{+}, D^{*-} \pi^{+}$and $D^{-} \rho^{+}$candidates using the same event shape criteria. Neither mixing-induced nor direct $C P$-violating asymmetry is observed as expected. As an additional test of the consistency of the background treatment, we add events from the $B^{0} \rightarrow \pi^{+} \pi^{-}$sideband and adjust their $\Delta E$ and $M_{\mathrm{bc}}$ values. A fit to this background-enriched control sample, which has a background fraction comparable to the $B^{0} \rightarrow \pi^{+} \pi^{-}$sample, yields $\mathcal{S}=0.08 \pm 0.06$ and $\mathcal{A}=0.03 \pm 0.04$, both consistent with a null asymmetry.

We determine the statistical significance from the likelihood function, taking into account the boundary of the physical region as well as the effect of the systematic error. The FeldmanCousins frequentist approach [14] gives a 99.6\% confidence level (C.L.) for $-1 \leq \mathcal{S}_{\pi \pi}<0$, equivalent to a $2.9 \sigma$ significance for a Gaussian error. A similar analysis yields a significance of $2.9 \sigma$ for $0<\mathcal{A}_{\pi \pi} \leq 1$. The $95 \%$ C.L. intervals are found to be $-1.00 \leq \mathcal{S}_{\pi \pi}<-0.39$ and $+0.30<\mathcal{A}_{\pi \pi} \leq+1.00$, respectively [15].

In summary, we have measured the $C P$ violation parameters in $B^{0} \rightarrow \pi^{+} \pi^{-}$decay. Our result for $\mathcal{S}_{\pi \pi}$ indicates that mixing-induced $C P$ violation is large. The large $\mathcal{A}_{\pi \pi}$ term is an indication of direct $C P$ violation in $B$ meson decay, and suggests that there is a large hadronic phase and interference between the tree and penguin amplitudes [16]. In this case the precise determination of $\sin 2 \phi_{2}$ from $\mathcal{S}_{\pi \pi}$ requires additional measurements including the branching fractions for the decays $B^{0} \rightarrow \pi^{0} \pi^{0}$ [17].

We wish to thank the KEKB accelerator group for the excellent operation of the KEKB accelerator. We acknowledge support from the Ministry of Education, Culture, Sports, Science, and Technology of Japan and the Japan Society for the Promotion of Science; the Australian Research Council and the Australian Department of Industry, Science and Resources; the National Science Foundation of China under contract No. 10175071; the Department of Science and Technology of India; the BK21 program of the Ministry of Education of Korea and the CHEP SRC program of the Korea Science and Engineering Foundation; the Polish State Committee for Scientific Research under contract No. 2P03B 17017; the Ministry of Science and Technology of the Russian Federation; the Ministry of Education, Science and Sport of the Republic of Slovenia; the National Science Council and the Ministry of Education of Taiwan; and the U.S. Department of Energy. 
* on leave from Nova Gorica Polytechnic, Slovenia

$\dagger$ on leave from University of Toronto, Toronto ON

[1] M. Kobayashi and T. Maskawa, Prog. Theor. Phys. 49, 652 (1973).

[2] K. Abe et al. (Belle Collab.), Phys. Rev. Lett. 87, 091802 (2001); hep-ex/0202027, submitted to Phys. Rev. D.

[3] B. Aubert et al. (BaBar Collab.), Phys. Rev. Lett. 87, 091801 (2001); hep-ex/0201020, submitted to Phys. Rev. D.

[4] Throughout this Letter, the inclusion of the charge conjugate mode decay is implied unless otherwise stated.

[5] Another naming convention, $\beta\left(=\phi_{1}\right)$ and $\alpha\left(=\phi_{2}\right)$, is also used in the literature.

[6] A. B. Carter and A. I. Sanda, Phys. Rev. Lett. 45, 952 (1980); A. B. Carter and A. I. Sanda, Phys. Rev. D 23, 1567 (1981); I. I. Bigi and A. I. Sanda, Nucl. Phys. 193, 85 (1981).

[7] A general review of the formalism is given in I.I. Bigi, V.A. Khoze, N.G. Uraltsev, and A.I. Sanda, "CP Violation" page 175, ed. C. Jarlskog, World Scientific, Singapore (1989).

[8] M. Gronau, Phys. Rev. Lett. 63, 1451 (1989); D. London and R. Peccei, Phys. Lett. B 223, 257 (1989); M. Beneke, G. Buchalla, M. Neubert and C. T. Sachrajda, Nucl. Phys. B 606, 245 (2001); Y. Y. Keum, H-N. Li and A. I. Sanda, Phys. Rev. D 63, 054008 (2001); M. Ciuchini et al., Phys. Lett. B 515, 33 (2001); M. Gronau and J. L. Rosner, Phys. Rev. D 65, 013004 (2002).

[9] E. Kikutani ed., KEK Preprint 2001-157 (2001), to appear in Nucl. Instr. and Meth. A.

[10] A. Abashian et al. (Belle Collab.), Nucl. Instr. and Meth. A 479, 117 (2002).

[11] K. Abe et al. (Belle Collab.), Phys. Rev. Lett. 87, 101801 (2001); a Phys. Rev. D paper is in preparation.

[12] The Fox-Wolfram moments were introduced in G. C. Fox and S. Wolfram, Phys. Rev. Lett. 41, 1581 (1978). The Fisher discriminant used by Belle is described in 11] and K. Abe et al. (Belle Collab.), Phys. Lett. B 511, 151 (2001).

[13] D. E. Groom et al. (Particle Data Group), Eur. Phys. J. C15, 1 (2000).

[14] G. J. Feldman and R. D. Cousins, Phys. Rev. D 57, 3873 (1998).

[15] An ensemble of MC pseudo-experiments indicates a $1.6 \%$ probability to measure $C P$ violation at or above the one we observe when the input values are $\mathcal{S}_{\pi \pi}=\mathcal{A}_{\pi \pi}=0$.

[16] $\mathcal{S}_{\pi \pi}=0.03_{-0.56}^{+0.53}$ (stat) \pm 0.11 (syst) and $C_{\pi \pi}=-0.25_{-0.47}^{+0.45}$ (stat) \pm 0.14 (syst) in B. Aubert et al. (BaBar Collab.), Phys. Rev. D 65, 051502R (2002). Note that the sign convention for the coefficient of the cosine term $C_{\pi \pi}$ defined in the above reference is opposite to our convention, i.e. $C_{\pi \pi}=-\mathcal{A}_{\pi \pi}$.

[17] M. Gronau and D. London, Phys. Rev. Lett. 65, 3381 (1990); M. Gronau, D. London, N. Sinha and R. Sinha, Phys. Lett. B 514, 315 (2001). 